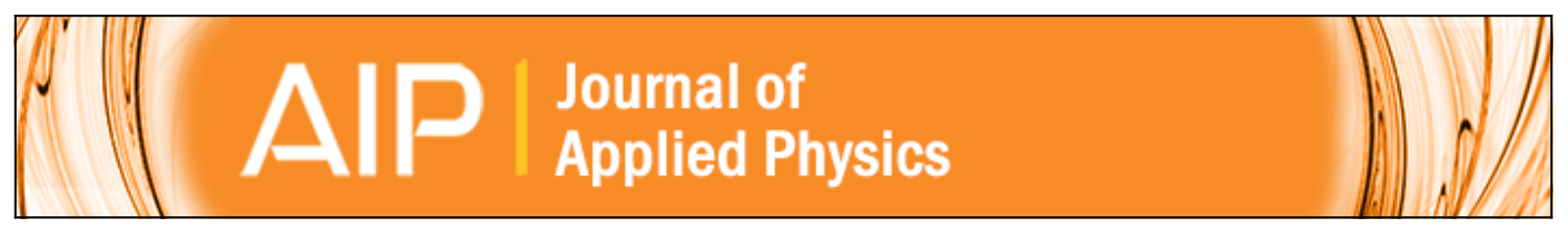

\title{
Interface modified thermally stable hole transporting layer for efficient organic light emitting diodes
}

Rakhi Grover, Ritu Srivastava, Janardan Dagar, M. N. Kamalasanan, and D. S. Mehta

Citation: Journal of Applied Physics 116, 063102 (2014); doi: 10.1063/1.4892396

View online: http://dx.doi.org/10.1063/1.4892396

View Table of Contents: http://scitation.aip.org/content/aip/journal/jap/116/6?ver=pdfcov

Published by the AIP Publishing

\section{Articles you may be interested in}

Investigation of hole injection enhancement by $\mathrm{MoO} 3$ buffer layer in organic light emitting diodes J. Appl. Phys. 114, 244505 (2013); 10.1063/1.4852835

Organic light emitting diodes using $\mathrm{NaCl}$ : N , N ' -bis(naphthalene-1-yl)- N, N ' -bis(phenyl)benzidine composite as a hole injection buffer layer

J. Appl. Phys. 108, 103703 (2010); 10.1063/1.3509150

Enhanced hole injection in organic light-emitting devices by using $\mathrm{Fe} 3 \mathrm{O} 4$ as an anodic buffer layer Appl. Phys. Lett. 94, 223306 (2009); 10.1063/1.3148657

Fullerene-doped hole transport molecular films for organic light-emitting diodes Appl. Phys. Lett. 86, 143509 (2005); 10.1063/1.1899241

Space-charge-limited charge injection from indium tin oxide into a starburst amine and its implications for organic light-emitting diodes

Appl. Phys. Lett. 72, 2448 (1998); 10.1063/1.121392

Frustrated by

old technology?

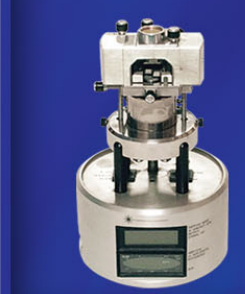

Is your AFM dead

and can't be repaired?

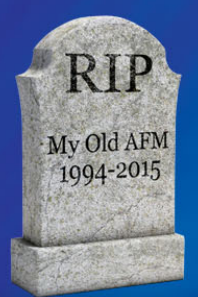

Sick of bad customer support?

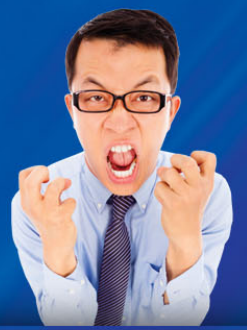

It is time to upgrade your AFM

Minimum $\$ 20,000$ trade-in discount for purchases before August 31st

Asylum Research is today's technology leader in AFM 


\title{
Interface modified thermally stable hole transporting layer for efficient organic light emitting diodes
}

\author{
Rakhi Grover, ${ }^{1, a)}$ Ritu Srivastava, ${ }^{2, b)}$ Janardan Dagar, ${ }^{2}$ M. N. Kamalasanan, ${ }^{2}$ \\ and D. S. Mehta ${ }^{3}$ \\ ${ }^{1}$ Amity Institute of Advanced Research and Studies (Materials and Devices), Amity University, Noida, \\ Uttar Pradesh 201303, India \\ ${ }^{2}$ Physics of Energy Harvesting Division, CSIR-National Physical Laboratory, CSIR-Network of Institute for \\ Solar Energy (NISE), Dr. K.S. Krishnan Road, New Delhi 110012, India \\ ${ }^{3}$ Department of Physics, Indian Institute of Technology Delhi, Hauz Khas, New Delhi 110016, India
}

(Received 31 October 2013; accepted 25 July 2014; published online 12 August 2014)

\begin{abstract}
Electrical transport in thermally stable 2, 7-bis [ $N, N$-bis (4-methoxy-phenyl) amino]-9, 9-spirobifluorene (MeO-Spiro-TPD) thin films has been investigated as a function of temperature and organic layer thickness. ITO/MeO-Spiro-TPD interface was found to be injection limited and has been studied in detail to find barrier height for hole injection. The thickness of tetrafluoro-tetracyano-quinodimethane thin films were optimized to be used as hole injection buffer layer which resulted in switching of charge transport mechanism from injection limited to space charge limited conduction above a critical thickness of $3 \mathrm{~nm}$. Hole mobility has been measured using transient space charge limited conduction (SCLC), field dependent SCLC, and top contact transistor characteristics. The charge carrier transport in interface modified hole only devices was analysed using Gaussian disorder model. The thermal stability of MeO-Spiro-TPD has been investigated by atomic force microscopy and X-ray diffraction studies. The study indicates a thermally stable and highly efficient hole transport material for application in organic semiconductor based devices. (C) 2014 AIP Publishing LLC. [http://dx.doi.org/10.1063/1.4892396]
\end{abstract}

\section{INTRODUCTION}

Efficiency and stability are two major concerns for organic light emitting diodes (OLEDs) to be adopted as reliable light sources. These two requirements are extensively dependent on the application of amorphous films of organic semiconductors with good charge transporting properties to function as electron or hole transport layers inside the devices. However, the organic materials having these desired characteristics should also have high thermal stability and proper energy level matching with the commonly used electrodes.

Hole transport materials (HTLs), $N, N^{\prime}$-Bis(3-methylphenyl)-(1,1'-biphenyl)-4,4'-diamine (TPD), $N, N^{\prime}$-diphenyl$N, N^{\prime}$-bis(1-naphthyl)-1,1'-biphenyl-4,4'-diamine $(\alpha-\mathrm{NPD})$, etc., are generally used due to their fairly large hole mobilities. ${ }^{1,2}$ However, these materials do not exhibit high thermal stability due to their low glass transition temperature $\left(T_{\mathrm{g}}\right)$ values, ${ }^{3}$ therefore, affecting overall device performance in terms of lifetime. In contrast to simple diamines, HTLs based on spiro concept have high $\mathrm{T}_{\mathrm{g}}$ values and therefore, higher thermal stability. ${ }^{4,5}$ In this concept, two similar charge transport molecules are joined through a spiro centre thereby increasing the steric demand of the resulting compound. In effect, the resulting compound has high $\mathrm{T}_{\mathrm{g}}$ value making it more thermally stable.

\footnotetext{
a) grover.rakhi@gmail.com

b) Author to whom correspondence should be addressed. Electronic mail: ritu@mail.nplindia.org.
}

2, 7-bis [ $N, N$-bis (4-methoxy-phenyl) amino]-9, 9spirobifluorene (MeO-Spiro-TPD) is known to be a spiro linked compound with a planarized configuration which can function as a transparent HTL. It exhibits high $\mathrm{T}_{\mathrm{g}}\left(115.7^{\circ} \mathrm{C}\right)$ and therefore high thermal stability. ${ }^{5,6}$ However, the charge carrier injection and transport studies in thin films of MeO-Spiro-TPD have not been systematically examined. Furthermore, the work function of commonly used anode ITO $(\sim 4.8 \mathrm{eV})$ is significantly lower than the HOMO of MeO-Spiro-TPD (5.21 eV). Therefore, the large injection barrier formed at ITO/HTL interface limits the direct application of this material in OLEDs. Several attempts have been made to reduce the hole injection barrier either by using interface layers (HIL); ${ }^{7,8}$ self-assembled monolayers or surface treatment of the electrode itself,, ${ }^{9,10}$ etc. Out of these techniques, insertion of thin layers of strong acceptor molecules like tetra-fluoro-tetracyano-quinodimethane $\left(\mathrm{F}_{4}\right.$-TCNQ) is a simple and efficient technique to enhance the hole injection from ITO to HTLs. ${ }^{11,12}$ However, ultra thin layer of $\mathrm{F}_{4}$-TCNQ as a HIL in combination with highly stable HTL, MeO-SpiroTPD has not been investigated earlier.

In the present report, the effect of thin films of $\mathrm{F}_{4}$-TCNQ (HIL) has been studied to reduce the hole injection barrier at the ITO/MeO-Spiro-TPD interface. The thickness of HIL has been optimized for reducing this barrier and to provide efficient hole injection into the highly stable HTL. The surface morphology and stability of thin films of MeO-Spiro-TPD have been studied using Atomic force microscope (AFM) and X-ray diffraction (XRD) studies. Charge carrier transport in interface modified thin films of MeO-Spiro-TPD has been investigated in detail at various thicknesses and temperatures 


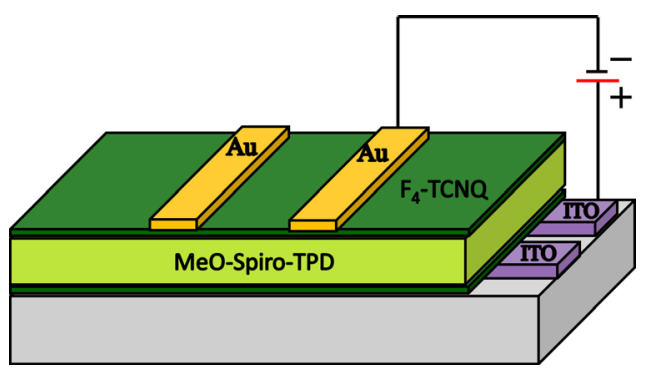

FIG. 1. Schematic device structure of interface modified hole only devices fabricated.

by fabricating hole only devices. Current density-voltage $(\mathrm{J}-\mathrm{V})$ characteristics of the hole only devices and top contact transistor characteristics have been used to extract hole mobility. The temperature dependency of charge carrier transport inside MeO-Spiro-TPD thin films has been further investigated using Gaussian disorder model (GDM). MeO-Spiro-TPD was found to be a thermally stable as well as efficient hole transport material which can be used efficiently in organic semiconductor based devices.

\section{EXPERIMENTAL}

ITO coated glass substrates having a sheet resistance of $20 \Omega / \mathrm{sq}$ and a thickness of $120 \mathrm{~nm}$ were used as the starting substrates. These glass plates were first patterned and cleaned by conventional solution cleaning process using deionized water, acetone, trichloroethylene, and isopropyl alcohol for 20 min each using an ultrasonic bath and were treated for $5 \mathrm{~min}$ with oxygen plasma. Thin films of MeO-SpiroTPD were deposited onto glass substrates under high vacuum $\left(\sim 10^{-6}\right.$ Torr) at a deposition rate of $0.2-0.3 \AA / \mathrm{s}$. Thickness of the deposited layers was measured in situ by a quartz crystal thickness monitor. Hole only devices were prepared in the configuration ITO/MeO-Spiro-TPD/Au with different thicknesses of MeO-Spiro-TPD thin films sandwiched in between high work function electrodes of ITO and Au. Different thicknesses of $\mathrm{F}_{4}$-TCNQ were inserted as HILs in between the electrodes and organic film. Fig. 1 shows the basic structure of these devices.

The active area of the devices was $4 \mathrm{~mm} \times 4 \mathrm{~mm}$. J-V measurements were performed in vacuum from room temperature to low temperature using a Keithley 2610 programmable digital source meter unit interfaced with computer and a cryostat (Advanced Research systems Inc., USA) in vacuum conditions. Organic field effect transistor (OFET) devices in the top contacts configuration were fabricated using

TABLE I. Physical and chemical properties of organic charge carrier injection and transport materials used in the present study.

\begin{tabular}{lcc}
\hline \hline Properties & $\begin{array}{c}\text { MeO-Spiro-TPD } \\
{[\text { Lum Tech. Corp] }}\end{array}$ & F4-TCNQ ${ }^{11}$ \\
\hline HOMO $(\mathrm{eV})$ & 5.21 & 8.34 \\
LUMO $(\mathrm{eV})$ & 2.33 & 5.24 \\
$\mathrm{~T}_{\mathrm{g}}\left({ }^{\circ} \mathrm{C}\right)$ & 115.7 & - \\
Mobility $\left(\mathrm{cm}^{2} / \mathrm{V} \mathrm{s}\right)$ & $8.87 \times 10^{-4}$ at $200 \mathrm{kV} / \mathrm{cm}$ (this work) & - \\
$(\mathrm{SCLC})$ & & \\
\hline \hline
\end{tabular}

the thermal evaporation method. A heavily doped $\mathrm{n}^{++}$type $\mathrm{Si}$ wafer with an oxide layer of $270 \mathrm{~nm}$ thickness $\left(\mathrm{C}=8 \times 10^{-9} \mathrm{~F} / \mathrm{cm}\right)$ was used as a substrate and was cleaned using solvents. Organic layer and gold electrodes were deposited at room temperature. The geometrical factors of the fabricated OFET devices were the Length $(\mathrm{L})=30 \mu \mathrm{m}$ and width $(\mathrm{W})=2 \mathrm{~mm}$ with the aspect ratio of order of 100 . Electrical measurements of fabricated OFET device were performed by Keithley 4200-interface with cascade probe station. Morphological analyses of MeO-Spiro-TPD thin films were performed using AFM technique (NTMDT Solver-Pro operated in tapping mode). Structural characterisation of these films was carried out by analysis of the X-ray diffraction (XRD) pattern using a Bruker AXS D8 advance diffractometer with $\mathrm{Cu} \mathrm{K} \alpha 1$ radiation $(\lambda=1.54056 \AA$ ).

\section{RESULTS AND DISCUSSIONS}

Table I shows the physical and chemical properties of the materials used in the present study. High work function materials, ITO $(4.8 \mathrm{eV})$ and gold $(5.2 \mathrm{eV})$ were used as hole injecting electrodes. The LUMO and HOMO levels of MeOSpiro-TPD (Lum. Tech. Corp.) are reported to be $2.33 \mathrm{eV}$ and $5.21 \mathrm{eV}$. Therefore, the injection of holes is predominantly more favourable than that of electrons in the device. The current observed in the devices with the configuration ITO/MeO-Spiro-TPD/Au would therefore be due to holes and the devices can be treated as hole only devices.

As shown in Fig. 2, there exists a small but significant injection barrier for the injection of holes as depicted by the energy offsets between ITO and MeO-Spiro-TPD. Fig. 3 shows the current density vs voltage $(\mathrm{J}-\mathrm{V})$ characteristics of hole only devices of MeO-Spiro-TPD for a fixed thickness of $300 \mathrm{~nm}$ and at different temperatures. The J-V curves clearly exhibited non linear behaviour with $\mathrm{J}$ decreasing substantially with decrease in temperature.

The charge transport in organic semiconductors can be either bulk limited or injection limited. Fowler-Nordheim (FN) type of tunnelling mechanism for the injection limited

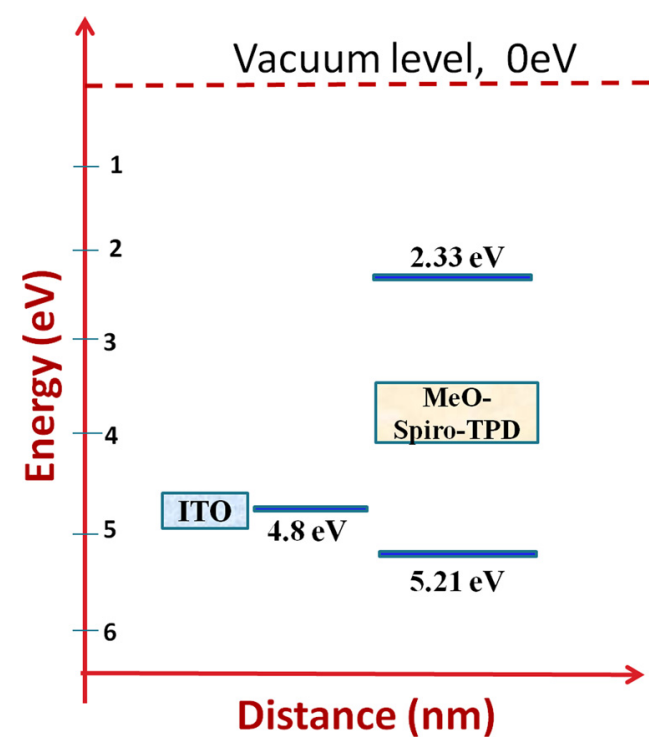

FIG. 2. Energy level diagram of ITO and MeO-Spiro-TPD. 


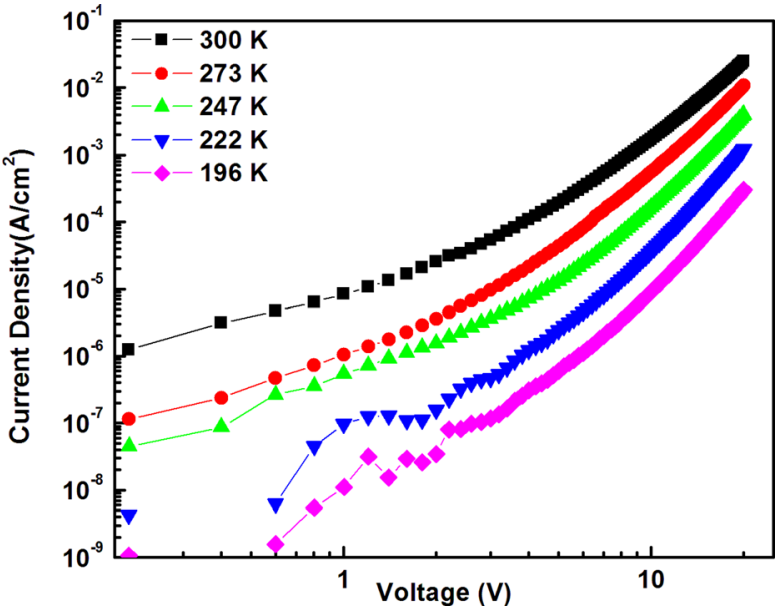

FIG. 3. J-V characteristics of ITO/MeO-Spiro-TPD $(300 \mathrm{~nm}) / \mathrm{Au}$ hole only device at different temperatures.

charge carrier conduction process was not expected in the present case because of the considerable temperature dependence of the J-V curves. The significant temperature dependence suggested thermionic type of injection from Fermi level of ITO to HOMO level of MeO-Spiro-TPD. Therefore, Richardson Schottky (RS) thermionic emission mechanism was employed for further studies. According to this model, current density $\mathrm{J}$ varies with applied field as ${ }^{13}$

$$
J=A^{*} T^{2} \exp \left(\frac{-\left(\phi_{b}-\sqrt{\frac{q F}{4 \pi \varepsilon \varepsilon_{0}}}\right)}{k_{b} T}\right),
$$

where A is the Richardson constant, $\mathrm{T}$ is the absolute temperature, $\phi_{b}$ is the barrier height, and $\mathrm{k}_{\mathrm{b}}$ is the Boltzmann constant. Fig. 4(a) shows ln (J) vs sqrt (F) curves for $300 \mathrm{~nm}$ thick MeO-Spiro-TPD film for different temperatures showing the RS type injection mechanism.

The plots in Fig. 4(a) exhibited linear dependence for all the temperatures further verifying the thermionic type of injection of charge carriers. These curves have been analysed in detail in order to estimate the barrier height of holes for the ITO/MeO-Spiro-TPD interface. The zero field current density $\left(\mathrm{J}_{\mathrm{o}}\right)$ has been obtained by extrapolating the $\ln (\mathrm{J})$ values at different temperatures to zero fields. Fig. 4(b) shows $\ln \left(\mathrm{J}_{\mathrm{O}} / \mathrm{T}^{2}\right)$ vs. $1 / \mathrm{T}$ plot, which gives a straight line with a slope of $-\left(\Phi_{\mathrm{b}} / \mathrm{k}_{\mathrm{b}}\right)$. This graph was used to calculate barrier height

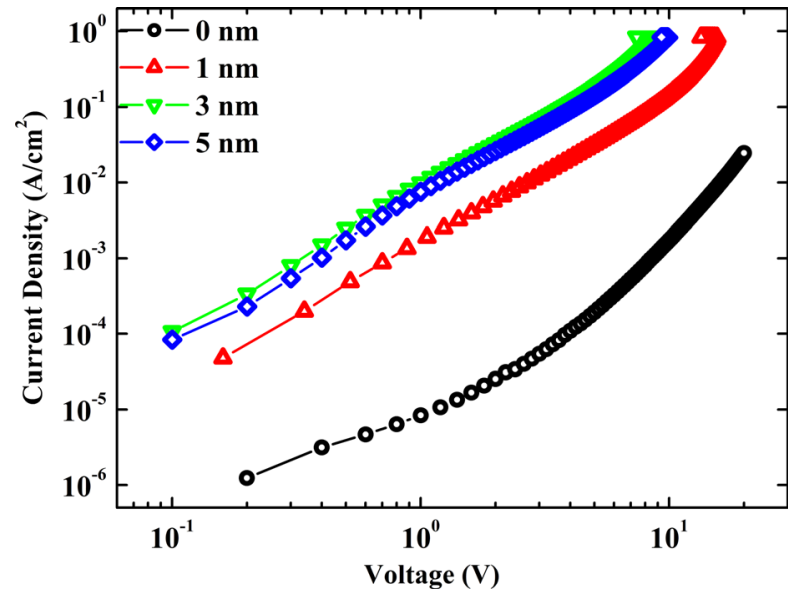

FIG. 5. J-V characteristics of interface modified hole only devices with different thicknesses of $\mathrm{F}_{4}$-TCNQ and fixed $300 \mathrm{~nm}$ thickness of MeO-SpiroTPD.

for holes at ITO/MeO-Spiro-TPD interface and was found to be $0.324 \mathrm{eV}$. In order to study the bulk properties, it is necessary to have ohmic injection at the electrode/organic interface to supply unhindered supply of charge carriers.

Therefore, to construct an ohmic contact between the HOMO of MeO-Spiro-TPD and Fermi level of ITO, the thickness of the well known ultrathin buffer layer of $\mathrm{F}_{4}$-TCNQ has been optimized to maximize the injection of holes. Fig. 5 shows the $\mathrm{J}-\mathrm{V}$ characteristics of interface modified hole only devices with the device structure $\mathrm{ITO} / \mathrm{F}_{4}-\mathrm{TCNQ} / \mathrm{MeO}-$ Spiro-TPD $/ \mathrm{F}_{4}-\mathrm{TCNQ} / \mathrm{Au}$ for different thicknesses of $\mathrm{F}_{4^{-}}$ TCNQ and fixed $300 \mathrm{~nm}$ thickness of MeO-Spiro-TPD thin film.

The current density was found to increase for all voltages, as the thickness of ultrathin $\mathrm{F}_{4}$-TCNQ layer increased. However, the enhancement saturated after a thickness $3 \mathrm{~nm}$ of $\mathrm{F}_{4}$-TCNQ film. This enhancement can be understood as the modification of the metal work function due to interaction with the thin layer of $\mathrm{F}_{4}$-TCNQ. The energy level alignment of vacuum sublimed $\mathrm{F}_{4}$-TCNQ films on various metal substrates has earlier been explained by an integer charge transfer (ICT) model. ${ }^{11}$ According to this model, as shown in Fig. 6, if the work function of metal is lower than the energy of the negative polaron state of organic material, electrons begin to flow from the metal to the organic at the interface when brought closer to each other. In this way, metal Fermi level is pinned to negative polaron state of the organic (a)

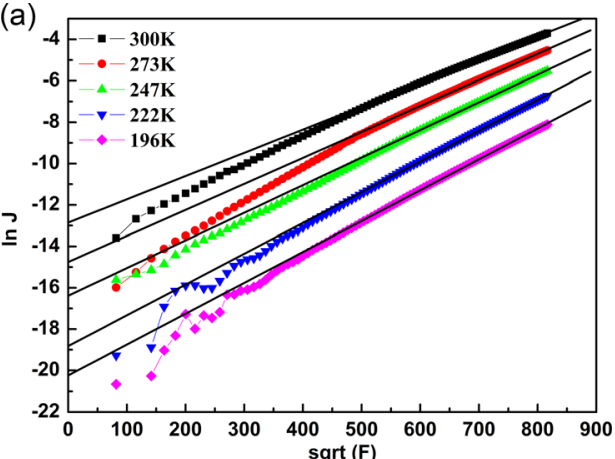

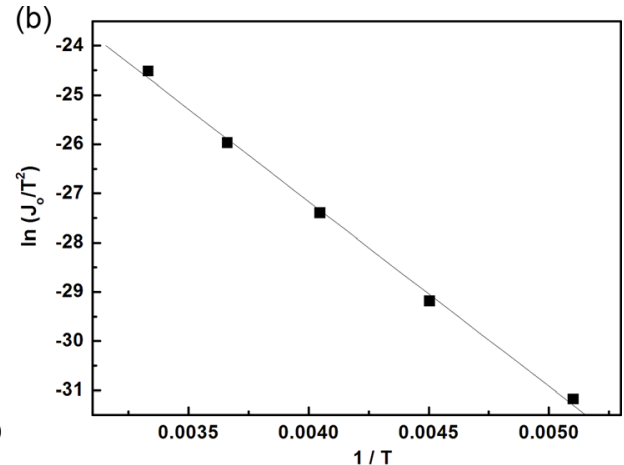

FIG. 4. (a) $\ln (\mathrm{J})$ vs. sqrt (F) and (b) $\ln \left(\mathrm{J}_{0} / \mathrm{T}^{2}\right)$ vs. $1 / \mathrm{T}$ characteristics of $\mathrm{ITO} / \mathrm{MeO}$-Spiro-TPD $\quad(300 \mathrm{~nm}) / \mathrm{Au}$ hole only device showing RS type injection mechanism. 


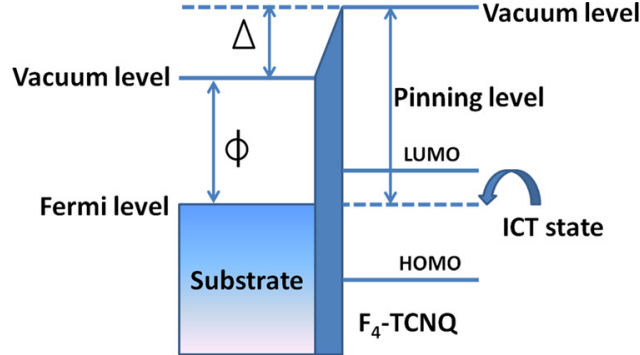

FIG. 6. Schematic energy level diagram of substrate/ $\mathrm{F}_{4}-\mathrm{TCNQ}$ interface showing the Fermi level pinning of the substrate to the LUMO of $\mathrm{F}_{4}$ TCNQ. ${ }^{11}$

material. Along the same context, a thin $\mathrm{F}_{4}$-TCNQ film is known to enhance the work function of most of the metals and this enhancement is dependent on the thickness of $\mathrm{F}_{4^{-}}$TCNQ layer. ${ }^{14}$

According to ICT model when $\mathrm{F}_{4}$-TCNQ is deposited on ITO $(4.8 \mathrm{eV})$, the Fermi level of the ITO is pinned to the charge transfer states of $\mathrm{F}_{4}$-TCNQ $(5.25 \mathrm{eV})$. Thus, after depositing a thin layer of $\mathrm{F}_{4}$-TCNQ, it act as virtual electrode and substrate work function is modified up to $\sim 5.25 \mathrm{eV}$.

Since the HOMO level of MeO-Spiro-TPD is $5.21 \mathrm{eV}$, the modified work function after the insertion of $\mathrm{F}_{4}$-TCNQ as a HIL is supposed to make the contact ohmic for holes with MeO-Spiro-TPD as HTL.

The ohmic contact at the interface between $\mathrm{ITO} / \mathrm{F}_{4^{-}}$ TCNQ and MeO-Spiro-TPD was verified by employing transient space charge-limited current measurements. Hole-only devices were fabricated with the structures ITO/MeO-Spiro$\mathrm{TPD} / \mathrm{Au}$ and ITO/F $4^{-T C N Q}(3 \mathrm{~nm}) / \mathrm{MeO}-\mathrm{Spiro}-\mathrm{TPD} / \mathrm{F}_{4^{-}}$ TCNQ (3 nm)/Au. A positively biased rectangular pulse was applied to this device using the function generator and monitored by the cathode ray oscilloscope (CRO). Charges travel across the sample and the space charge limited current was monitored by CRO. Transient space charge limited conduction (SCLC) graphs of MeO-Spiro-TPD with and without HIL at $20 \mathrm{~V}$ bias $\left(6.67 \times 10^{5} \mathrm{~V} / \mathrm{cm}\right.$ internal field $)$ are shown in Fig. 7 .

The device with a $\mathrm{F}_{4}$-TCNQ buffer layer shows a typical peak and subsequent steady-state current in the graph which is not observed in the device without this buffer layer. This confirmed the ohmic nature of the $\mathrm{ITO} / \mathrm{F}_{4}-\mathrm{TCNQ} / \mathrm{MeO}-$ Spiro-TPD contact. ${ }^{15}$ The fastest response time $\tau$ was determined from transient plot and the carrier mobility was evaluated from the relation

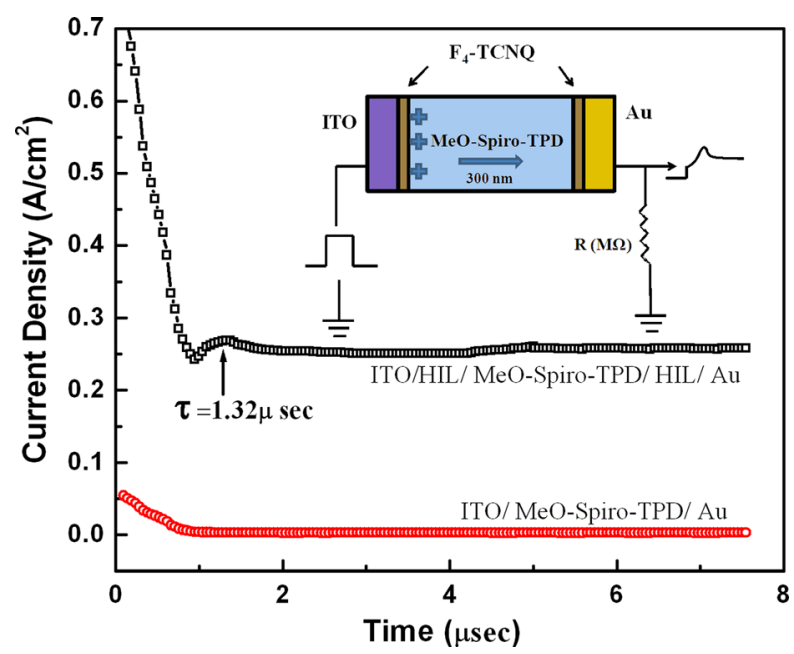

FIG. 7. Transient SCLC signals observed from devices with structures of $\mathrm{ITO} / \mathrm{F}_{4}$-TCNQ $(3 \mathrm{~nm}) / \mathrm{MeO}-\mathrm{Spiro}-\mathrm{TPD} / \mathrm{F}_{4}-\mathrm{TCNQ}(3 \mathrm{~nm}) / \mathrm{Au}$ and ITO/MeOSpiro-TPD/Au at $20 \mathrm{~V}$.

$$
\mu=\frac{0.787 d^{2}}{\tau \cdot V}
$$

where $\mathrm{d}$ is the film thickness and $\mathrm{V}$ is the applied voltage. The typical value of the mobility was found to be $2.68 \times 10^{-5} \mathrm{~cm}^{2} / \mathrm{V} \mathrm{s}$. In order to confirm the ohmic nature of the ITO/F $/ \mathrm{F}_{4}$ TCNQ/MeO-Spiro-TPD contact, J-V characteristics of the interface modified devices were studied. Fig. 8(a) shows the J-V characteristics of hole only devices with the device structure ITO/F $/ \mathrm{F}_{4}$-TCNQ/ MeO-Spiro-TPD/F 4 -TCNQ/ $\mathrm{Au}$ for different thicknesses of MeO-Spiro-TPD and fixed $3 \mathrm{~nm}$ thickness of $\mathrm{F}_{4}$-TCNQ as the HIL.

The current density was found to be proportional to the square of the applied voltage and this feature was observed for all sample thicknesses. The interface modified devices exhibit space charge limited conduction, i.e., the current density was found to be square of the applied voltage. This indicates that a thin layer of $\mathrm{F}_{4}$-TCNQ makes the contact ohmic which leads to SCLC in MeO-Spiro-TPD thin films. As shown in the inset of Fig. 8(a), the current density in forward and reverse directions was found to be similar signifying negligible built in potential in the devices. The relation between current density (J) and voltage (V) for SCLC with field independent mobility $(\mu)$ is given by the Mott Gurney equation $^{16}$
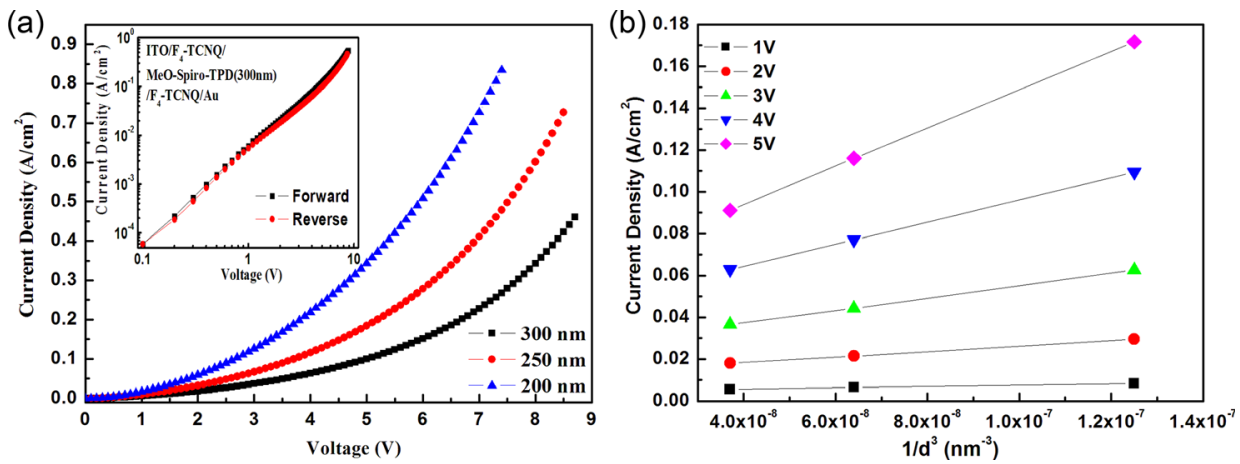

FIG. 8. (a) J-V and (b) $1 / \mathrm{d}^{3}$ characteristics of interface modified hole only devices with different thicknesses of $\mathrm{MeO}-$ Spiro-TPD and fixed $3 \mathrm{~nm}$ thicknesses of $\mathrm{F}_{4}$-TCNQ. Inset of (a) shows the $\mathrm{J}-\mathrm{V}$ characteristics for the device with $300 \mathrm{~nm}$ thick MeO-Spiro-TPD film in forward and reverse directions. 


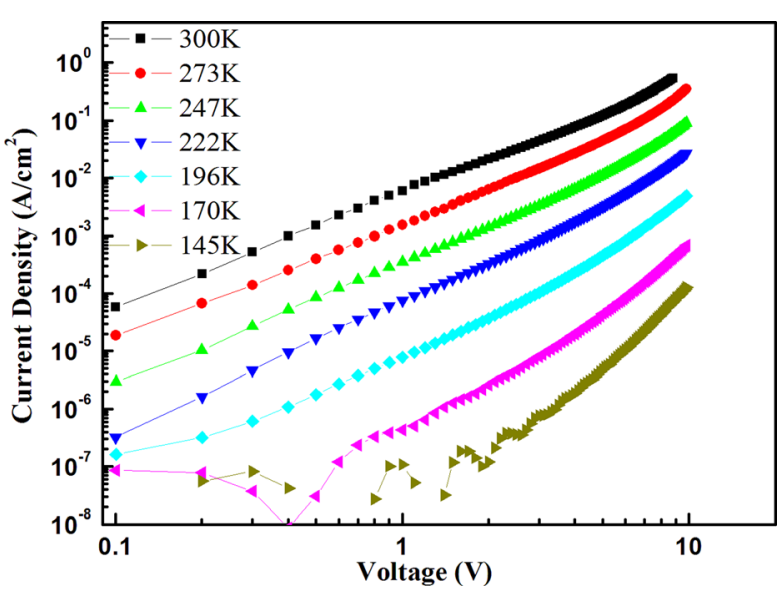

FIG. 9. J-V characteristics of interface modified hole only devices as a function of temperature.

$$
J=\frac{9}{8} \varepsilon \varepsilon_{0} \frac{V^{2}}{d^{3}} \mu,
$$

where $\varepsilon$ is the dielectric constant taken as 3 for organic semiconductors, ${ }^{17} \varepsilon_{0}$ is the permittivity of free space, $\mu$ is the mobility of holes, and $d$ is the thickness of the film. The observed thickness dependence of the current density is shown in Fig. 8(b). This indicated that the conduction process involves bulk of the material and the interface effects are negligible. The effective charge carrier mobility from SCLC region was calculated using Eq. (3) and was found to be $8.87 \times 10^{-4} \mathrm{~cm}^{2} / \mathrm{V} \mathrm{s}$ at a field of $200 \mathrm{kV} / \mathrm{cm}$. For higher voltages, the increase in current density was found to be steeper than as expected by SCLC equation. Such steeper enhancement in current density is generally known to be due to enhanced mobility of charge carriers with the applied higher electric field values as given by Murgatroyed: ${ }^{18}$

$$
J(F, T)=\frac{9}{8} \varepsilon \varepsilon_{0} \frac{V^{2}}{d^{3}} \mu(0, T)(0.89 \gamma \sqrt{F}),
$$

where $\varepsilon$ is the dielectric constant taken as $3, \varepsilon_{0}$ is the permittivity of free space, $\mu(0, \mathrm{~T})$ is the temperature dependent zero field mobility of holes (further called as $\mu_{0}$ ), $\gamma$ is field lowering constant, $d$ is thickness of the film, and $F=V / d$ is the electric field. Furthermore, the charge carrier conduction mechanism in disordered organic thin films is also thermally activated. The J-V characteristics of the interface modified hole only devices have therefore been studied as a function of temperature also as shown in Fig. 9.

The current density varied significantly with the voltage as well as temperature. These graphs were analysed in detail using Eq. (4). Fig. 10 shows the experimentally obtained temperature dependent $\mathrm{J}-\mathrm{V}$ curves for the device structure

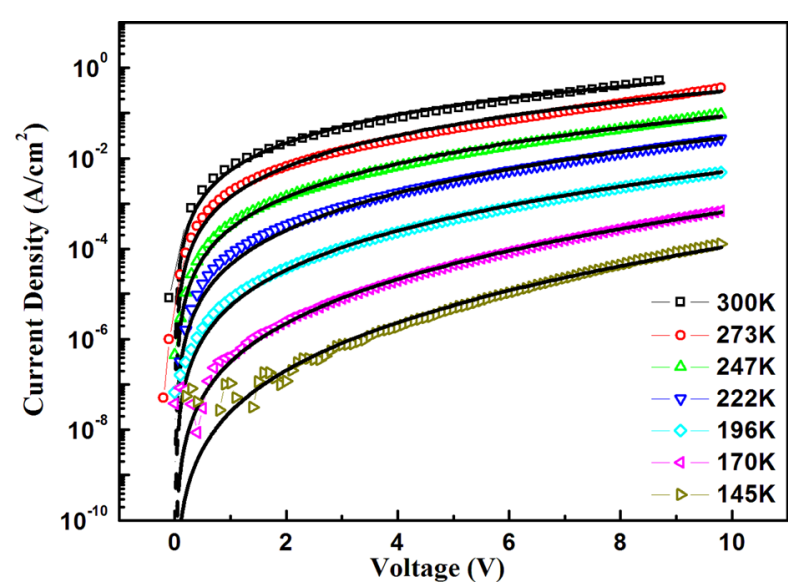

FIG. 10. Theoretical fitted J-V characteristics at various temperature of interface modified hole only device, symbols represent the experimental values and the solid lines represent the theoretical fits.

ITO/F 4 -TCNQ (3 nm)/MeO-Spiro-TPD (300 nm)/F 4 -TCNQ $(3 \mathrm{~nm}) / \mathrm{Au}$ along with the numerically fitted $\mathrm{J}-\mathrm{V}$ curves using $\mu_{0}$ and $\gamma$ as the fitting parameters for each temperature. Reasonably good fitting of the experimental J-V curves was obtained for each temperature and for higher voltages.

The current density characteristics were found to be strongly temperature dependent and exhibited substantial decrease in current density values as the temperature decreased. The values of $\mu_{0}(\mathrm{~T})$ and $\gamma$ used for fitting at different temperature have been summarised in Table II.

However, pure SCLC mechanism is based on weak dependence of current density on temperature. Therefore, for observed temperature dependence, the SCLC type of conduction can be better described by incorporating field and temperature dependence of the mobility.

In addition, charge carrier transport properties of organic semiconductors are better described by hopping transport mechanism dependent on the energetic and positional disorder present in the disordered films. GDM explained the charge carrier transport in organic semiconductors as a biased random walk among the hopping sites having randomly distributed site energies which leads to the mobility of the form: ${ }^{19}$

$$
\begin{aligned}
\mu(F, T)= & \mu_{\infty} \exp \left(-\left(\frac{2 \sigma}{3 K_{b} T}\right)^{2}\right) \\
& \times \exp \left(C\left\{\left(\frac{\sigma}{K_{b} T}\right)^{2}-\Sigma^{2}\right\} \sqrt{F}\right)
\end{aligned}
$$

where the terms $\sigma, \Sigma, \mathrm{C}$, and $\mu_{\infty}$ are defined as the energetic disorder, positional disorder, specific parameter depending on the intersite distance, and high temperature limit of mobility,

TABLE II. Values of $\mu_{0}$ and $\gamma$ at different temperatures for ITO/MeO-Spiro-TPD film interface modified by $\mathrm{F}_{4}$-TCNQ.

\begin{tabular}{lccccc}
\hline \hline Parameter & \multicolumn{5}{c}{ ITO/F - TCNQ $(3 \mathrm{~nm}) / \mathrm{MeO}-$ Spiro-TPD $(30 \mathrm{~nm}) / \mathrm{F}_{4}-\mathrm{TCNQ}(3 \mathrm{~nm}) / \mathrm{Au}$} \\
\hline Temperature & $300 \mathrm{~K}(\mathrm{RT})$ & $273 \mathrm{~K}$ & $247 \mathrm{~K}$ & $222 \mathrm{~K}$ & $196 \mathrm{~K}$ \\
$\mu_{0}\left(\mathrm{~cm}^{2} / \mathrm{V} \mathrm{s}\right)$ & $2.7 \times 10^{-4}$ & $5 \times 10^{-5}$ & $8.5 \times 10^{-6}$ & $9.6 \times 10^{-7}$ & $1 \times 10^{-7}$ \\
$\gamma(\mathrm{cm} / \mathrm{V})^{1 / 2}$ & $9.6 \times 10^{-4}$ & $2 \times 10^{-3}$ & $3 \times 10^{-3}$ & $4 \times 10^{-3}$ & $5.9 \times 10^{-3}$ \\
\hline \hline
\end{tabular}



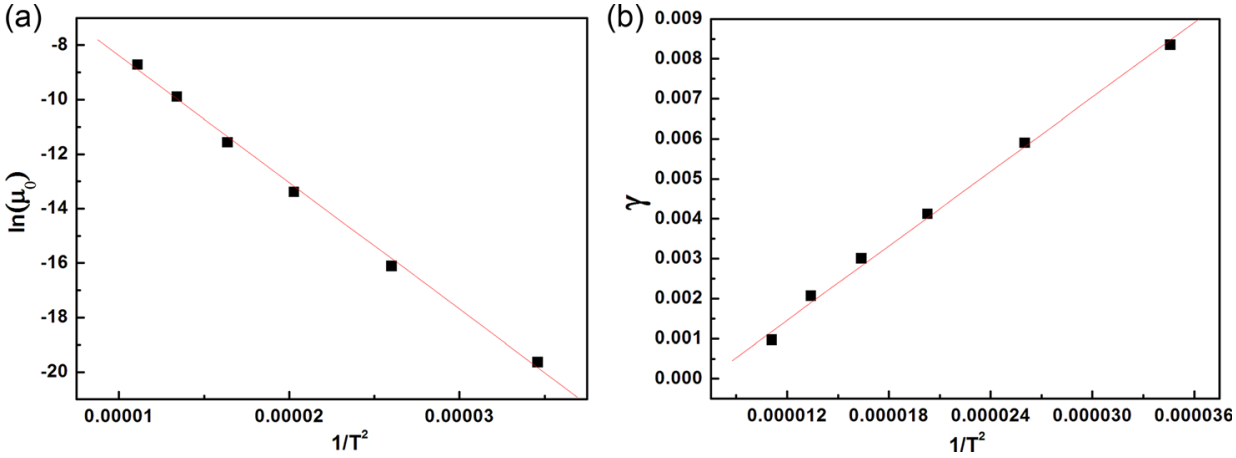

FIG. 11. Variation of (a) zero field mobility $\left(\mu_{0}\right)$ and $(\mathrm{b})$ field lowering factor $(\gamma)$ with $1 / \mathrm{T}^{2}$ for MeO-Spiro-TPD interface modified hole only devices.

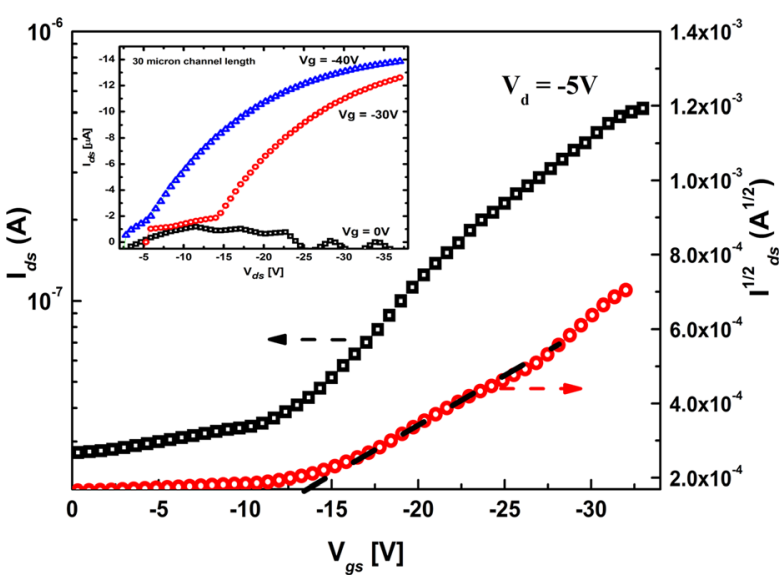

FIG. 12. $I_{\mathrm{ds}}$ vs. $\mathrm{V}_{\mathrm{gs}}$ and $\sqrt{I_{d s}}$ vs. $\mathrm{V}_{\mathrm{gs}}$ curves for the OTFTs fabricated. Inset shows the $I_{d s}$ vs $V_{d s}$ output characteristics. respectively. These parameters can be calculated from the plots of $\ln (\mu(0, \mathrm{~T}))$ and $\gamma$ vs $1 / \mathrm{T}^{2}$ as shown in Fig. 11.

Using Eq. (5), the obtained GDM parameters are $\mu_{\infty}=2.3 \times 10^{-2} \mathrm{~cm}^{2} / \mathrm{V} \quad \mathrm{s}, \quad \sigma=88.28 \mathrm{meV}, \mathrm{C}=2.9 \times 10^{-4}$ $(\mathrm{cm} / \mathrm{V})^{1 / 2}, \Sigma=2.74$. The values of all these parameters were found to be in the typical range for a whole class of disordered molecular solids. ${ }^{20}$

OFETs were fabricated in the top contact configuration. The electrical characteristics of the OFETs are given in Fig. 12.

The drain current $\mathrm{I}_{\mathrm{ds}}$ increases as the negative gate voltage $V_{g}$ values are increased. The sign of the field enhanced current $\left(\mathrm{I}_{\mathrm{ds}}<0\right.$ with $\left.\mathrm{V}_{\mathrm{g}}<0\right)$ is consistent with the $\mathrm{p}$ type behaviour of $\mathrm{MeO}$-Spiro-TPD in the accumulation regime of operation of the device. The threshold voltage was found to be $-13.5 \mathrm{~V}$. The following conventional relation for mobility applied in inorganic FETs has been used in the present case: ${ }^{21}$
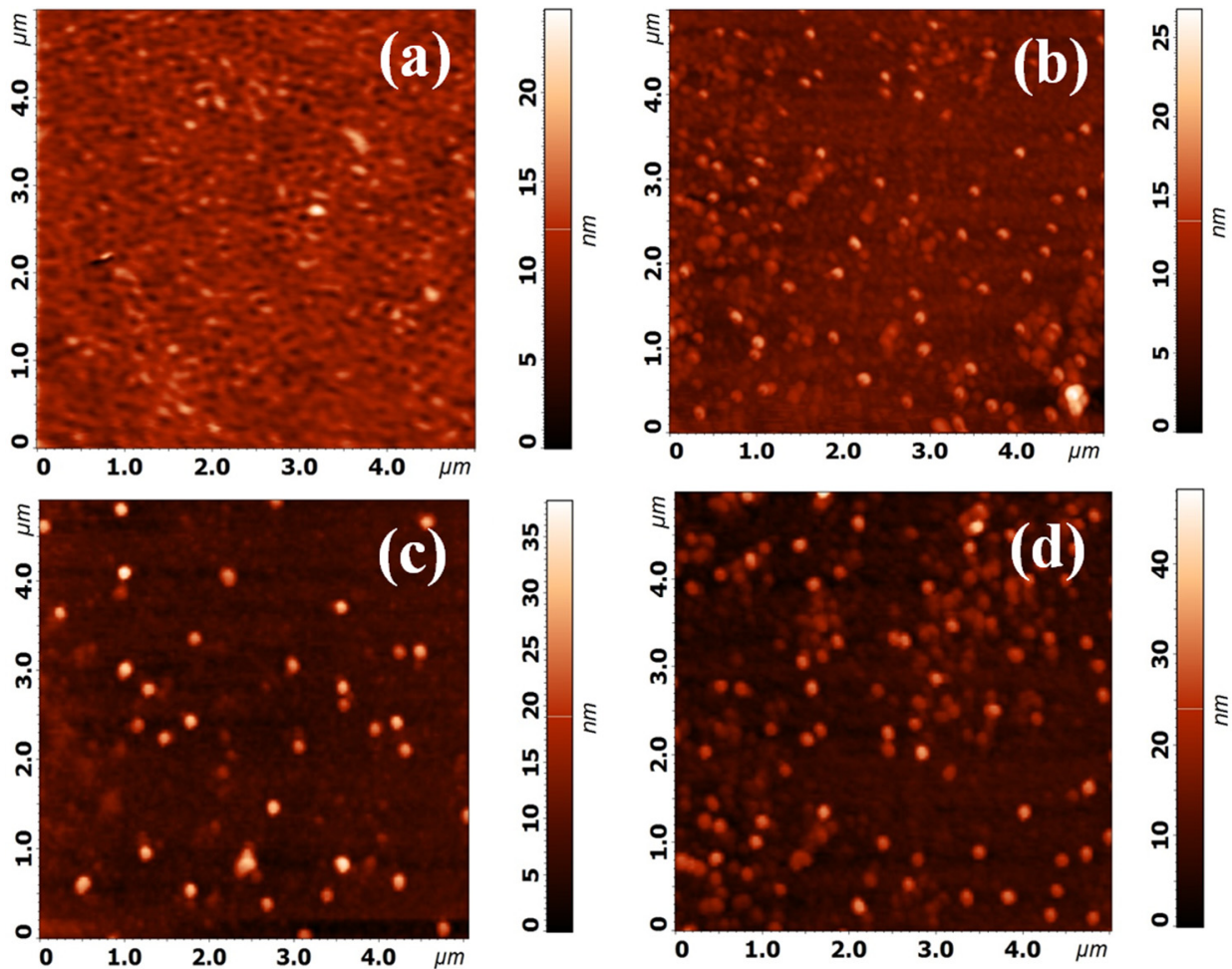

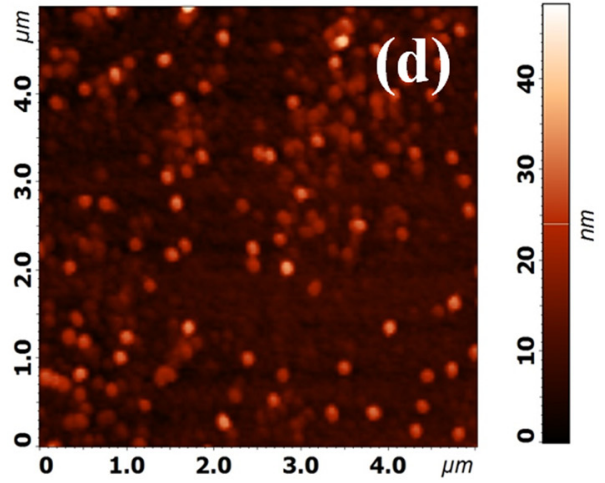

FIG. 13. AFM images of $50 \mathrm{~nm}$ thick films of MeO-Spiro-TPD annealed at (a) $0{ }^{\circ} \mathrm{C}$ (b) $50{ }^{\circ} \mathrm{C}$, (c) $70^{\circ} \mathrm{C}$, and (d) $90^{\circ} \mathrm{C}$ temperatures. 


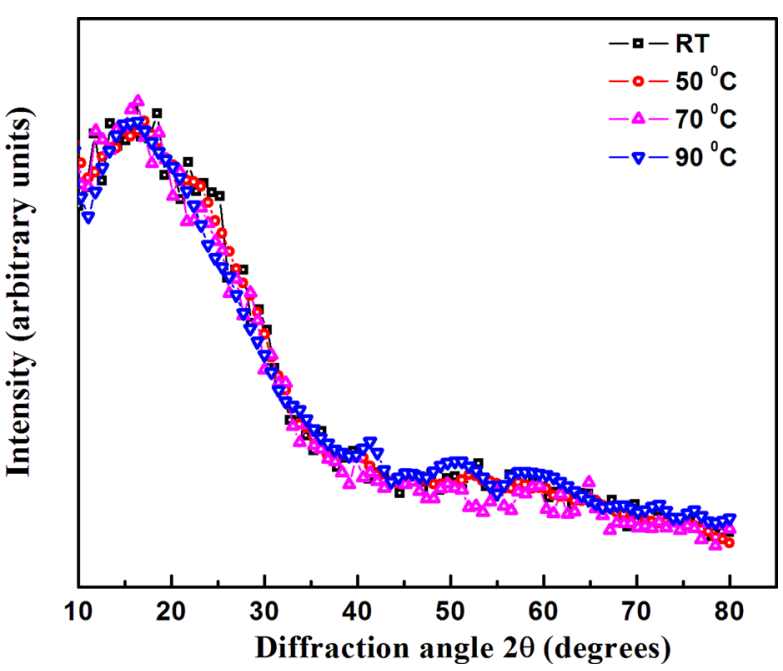

FIG. 14. XRD patterns of $50 \mathrm{~nm}$ thick films of MeO-Spiro-TPD on glass substrates annealed at $0{ }^{\circ} \mathrm{C}, 50^{\circ} \mathrm{C}, 70^{\circ} \mathrm{C}$, and $90^{\circ} \mathrm{C}$ temperatures.

$$
I_{d s}=\left(\frac{W}{2 L}\right) \mu C_{i}\left(V_{g}-V_{t h}\right)^{2},
$$

where $I_{d s}$ is the drain-source current in the saturation region, $\mu$ is the field-effect mobility, $\mathrm{L}$ and $\mathrm{W}$ are the channel length and width, respectively, $\mathrm{V}_{\mathrm{g}}$ and $\mathrm{V}_{\text {th }}$ are the gate voltage and threshold voltage, respectively, and $C_{i}$ is the capacitance of the insulating layer per unit area. The field-effect mobility in the saturation region, using this equation, has been estimated to be $1.93 \times 10^{-3} \mathrm{~cm}^{2} / \mathrm{V}$ s. An on/off ratio of $1.98 \times 10^{1}$ for $\mathrm{V}_{\mathrm{ds}}=-5 \mathrm{~V}$ could be obtained.

Apart from having high conductivity, these film also exhibit high morphological stability. The surface morphology of MeO-Spiro-TPD thin films was examined using AFM image analysis. The effect of elevated temperatures was studied to confirm the thermal stability of MeO- Spiro-TPD thin films. Figs. 13(a)-13(d) show the AFM images of $50 \mathrm{~nm}$ thick films of MeO-Spiro-TPD on glass substrates annealed at $0{ }^{\circ} \mathrm{C}, 50^{\circ} \mathrm{C}, 70^{\circ} \mathrm{C}$, and $90^{\circ} \mathrm{C}$ temperatures, respectively.

These films exhibited average roughness of $1.26 \mathrm{~nm}$, $1.42 \mathrm{~nm}, 2.18 \mathrm{~nm}$, and $2.65 \mathrm{~nm}$ for the as deposited film (Fig. 13(a)), after annealing at $50^{\circ} \mathrm{C}$ (Fig. 13(b)), $70^{\circ} \mathrm{C}$ (Fig. 13(c)) and $90^{\circ} \mathrm{C}$ (Fig. 13(d)) temperatures, respectively. Thin films of the material were found to be quite, dense, amorphous, and stable. The slight increase in average roughness values can be attributed to evolution of some nanosized crystallites in the film as the temperature was increased from $70^{\circ} \mathrm{C}$ to $90^{\circ} \mathrm{C}$. This type of nano-structural evolution in the films was further verified by X-ray diffraction studies. Fig. 14 shows the XRD patterns of $50 \mathrm{~nm}$ thick films of $\mathrm{MeO}-$ Spiro-TPD on glass substrates annealed at $0{ }^{\circ} \mathrm{C}, 50{ }^{\circ} \mathrm{C}$, $70^{\circ} \mathrm{C}$, and $90^{\circ} \mathrm{C}$ temperatures, respectively.

A freshly deposited $50 \mathrm{~nm}$ thick film of MeO-Spiro-TPD on glass substrate exhibited amorphous behaviour with no prominent peaks in the XRD pattern. Even as the film was annealed at $50^{\circ} \mathrm{C}, 70^{\circ} \mathrm{C}$, and $90^{\circ} \mathrm{C}$ temperatures, no appreciable differences were found in the patterns depicting thermal stability of the films. Therefore, thin films of
MeO-Spiro-TPD can be used as an efficient and stable material in organic semiconductor based devices.

\section{CONCLUSIONS}

Charge carrier injection and transport in thin films of thermally stable HTL, MeO-Spiro-TPD, have been investigated in detail. The thickness of F4 TCNQ as a HIL at the ITO/HTL interface was optimized to be $3 \mathrm{~nm}$ for maximum value of the current density making the contact ohmic. The $\mathrm{J}-\mathrm{V}$ characteristic with interface modification exhibit SCLC from which mobility of holes in MeO-Spiro-TPD thin films has been calculated which is very near to the field effect mobility. The mobility value obtained from DI SCLC experiments is lesser than the value obtained by steady state SCLC measurements. This difference in mobility values may be ascribed to the large energetic disorder parameter of the material, i.e., $88 \mathrm{meV}$ as calculated using the Gaussian disorder model. The temperature dependent $\mathrm{J}-\mathrm{V}$ characteristics of interface modified hole only devices have also been analysed in the framework of SCLC including a field and temperature dependent mobility. Thin films of MeO-Spiro-TPD were also found to be thermally stable using AFM and XRD studies. ITO/F4-TCNQ (3 nm)/MeO-Spiro-TPD was found to be a competent ITO/HIL/HTL system for efficient injection and transport of holes inside the device.

\section{ACKNOWLEDGMENTS}

The authors acknowledge the Council of Scientific \& Industrial Research (CSIR), New Delhi, India, for the research fellowship, Network Project 55 and the Project 03(1147)/09/EMR-II.

${ }^{1}$ D. F. O'Brien, P. E. Burrows, S. R. Forrest, B. E. Koene, D. E. Loy, and M. E. Thompson, Adv. Mater. 10, 1108 (1998).

${ }^{2}$ C. Adachi, T. Tsutsui, and S. Saito, Appl. Phys. Lett. 55, 1489 (1989).

${ }^{3}$ E. M. Han, L. M. Do, Y. Nidome, and M. Fujihira, Chem. Lett. 23, 969 (1994).

${ }^{4}$ U. Bach, D. Lupo, P. Comte, J. E. Moser, F. Weissörtel, J. Salbeck, H. Spreitzer, and M. Grätzel, Nature 395, 583 (1998).

${ }^{5}$ T. P. I. Saragi, "Organic Field Effect Transistors and Phototransistors based on amorphous materials," Ph.D. dissertation, Fachbereich Physik, Gesamthochschule-Universität Kassel, Kassel, Germany (2004).

${ }^{6}$ R. Grover, R. Srivastava, M. N. Kamalasanan, and D. S. Mehta, RSC Adv. 4, 10808 (2014)

${ }^{7}$ S. A. V. Slyke, C. H. Chen, and C. W. Tang, Appl. Phys. Lett. 69, 2160 (1996).

${ }^{8}$ S. A. Carter, M. Angelopoulos, S. Karg, P. J. Brock, and J. C. Scott, Appl. Phys. Lett. 70, 2067 (1997).

${ }^{9}$ K. H. Lee, H. W. Jang, K. B. Kim, Y. H. Tak, and J. L. Lee, J. Appl. Phys. 95, 586 (2004).

${ }^{10}$ Z. Z. You and J. Y. Dong, Appl. Surf. Sci. 249, 271 (2005).

${ }^{11}$ S. Braun and W. R. Salaneck, Chem. Phys. Lett. 438, 259 (2007).

${ }^{12}$ W. Gao and A. Kahn, Appl. Phys. Lett. 79, 4040 (2001).

${ }^{13}$ S. Miyata and H. S. Nalwa, Organic Electroluminescent Materials and Devices (Gordon and Breach Science Publishers, Amsterdam, 1997).

${ }^{14}$ O. Rana, R. Srivastava, G. Chauhan, M. Zulfequar, M. Husain, P. C. Srivastava, and M. N. Kamalasanan, Phys. Status Solidi A 209, 2539 (2012).

${ }^{15}$ S. C. Tse, S. W. Tsang, and S. K. So, J. Appl. Phys. 100, 063708 (2006).

${ }^{16}$ M. A. Lampert and P. Mark, Current Injection in Solids (Academic Press, New York, 1970).

${ }^{17}$ T. Y. Chu and O. K. Song, Appl. Phys. Lett. 90, 203512 (2007).

${ }^{18}$ P. N. Murgatroyd, J. Phys. D: Appl. Phys. 3, 151 (1970).

${ }^{19}$ H. Bassler, G. Herrmann, N. Riehl, and G. Vaubel, J. Phys. Chem. Solids 30, 1579 (1969).

${ }^{20}$ P. W. M. Blom, M. J. M. De Jong, and M. G. Van Munster, Phys. Rev. B 55, R656 (1997).

${ }^{21}$ J. Zaumseil and H. Sirringhaus, Chem. Rev. 107, 1296 (2007). 